\title{
Atuação da Fisioterapia na Reversão das Atelectasias: Um relato de caso na Unidade de Terapia Intensiva
}

\section{The Performance of the Physiotherapy in the Reversion of the Atel- ectasis: A report of a case in the Intensive Therapy Unit}

Cleize Silveira Cunha ${ }^{1}$

Rafael Vianna Toledo ${ }^{2}$

\begin{abstract}
Resumo
Esta pesquisa é descrita na inclusão de relato de caso clínico de um paciente ventilado mecanicamente. Este tipo de pesquisa foi escolhido pelos autores para que sirva como base de descrição à reversão da atelectasia ou colapso pulmonar, que é uma complicação respiratória decorrente da obstrução de um brônquio, ou pulmão, seja por secreção ou ainda objeto ou corpo sólido, impedindo desta forma a passagem do ar e levando à diminuição do número de alvéolos funcionantes. Três fatores combinados ou independentes que contribuem para o desenvolvimento de uma atelectasia: a força inadequada de distensão pulmonar; obstrução das vias aéreas e insuficiência no surfactante. São classificadas como obstrutivas, compressivas, por contração e focal. Os sinais e sintomas das atelectasias variam com o tamanho da área pulmonar envolvida, o estado prévio de saúde do paciente e a duração do problema. A dispnéia é o mais comum dos sintomas associados a atelectasias. O tratamento deverá ser escolhido de acordo com a causa da atelectasia, determinando assim, o objetivo para cada tipo, que inclui técnicas desobstrutivas e de reexpansão pulmonar, técnicas essas que melhoram significativamente a ventilação da área comprometida, proporcionando a reversão da atelectasia como foi comprovado nesta pesquisa.
\end{abstract}

\footnotetext{
1 Mestranda em Terapia Intensiva pela UFRJ

cleize.tavares@snt.com.br

2 Especialista em cárdio-respiratória

rafaeltoledo4@hotmail.com
} 
Abstract

This research is described in the inclusion of a report about a clinical case of a mechanically ventilated patient. This type of research was chosen by the authors so that it serves as a base of description to the reversion of the atelectasis or pulmonary collapse, that is a respiratory complication derived by the blockage of a bronchi, or lung, either by secretion or still object or a solid body, yet obstructing the air passing and taking to the reduction of the number of functional alveolus. Three agreed or independent factors that contribute in the development of an atelectasis: the inadequate strength of pulmonary dilation; the blockage of the aerial directions and insufficiency in the surfactant. They are classified as obstructive, compression, for contraction and focal. The signals and symptoms of the atelectasis vary with the size of the involved pulmonary area, the previous state of health of the patient and length of the problem. The dyspnea is the most common of the symptoms associated with the atelectasis. The treatment will have to be chosen in accordance with the cause of the atelectasis, thus, determining the objective for each type, that includes desobstruent techniques and of pulmonary reexpansion, techniques, yet, that significantly improves the ventilation of the compromised area, providing the reversion of the atelectasis as it was proven in this research.

Keywords: Physiotherapy, Treatment, Development. 


\section{Introdução}

A Atelectasia representa uma das principais indicações de fisioterapia em Unidades de Tratamento Intensivo (UTI), uma vez que pode conduzir o paciente a uma insuficiência respiratória importante afetando a troca gasosa nos mesmos. Existem inúmeros procedimentos utilizados no tratamento fisioterapêutico na atelectasia, em que podemos destacar técnicas de desobstrução e técnicas de reexpansão pulmonar.

O objetivo desta pesquisa é comprovar a eficácia do tratamento fisioterapêutico para a reversão da atelectasia em pacientes que estejam na UTI, e discutir informações sobre a doença e o tratamento adequado para cada tipo de paciente, avaliando a eficiência das técnicas aplicadas na fisioterapia. Consta neste trabalho uma revisão bibliográfica e fisioterapêutica.

Descrevemos em nosso estudo um relato de caso com paciente internado na Unidade de Terapia Intensiva do Hospital VITA - Volta Redonda - RJ, no dia 03 de julho de 2006, ocasião em que se observou a reversão da atelectasia total de pulmão esquerdo, com a utilização de técnicas de fisioterapia respiratória. Foram realizados dois atendimentos, quando a conduta fisioterapêutica teve como objetivo primordial a reexpansão pulmonar a fim de que dessa forma, se normalizasse o gradiente Ventilação-Perfusão (V/Q), destacam-se ainda, como outros objetivos, a eliminação das secreções e aumento da complacência pulmonar.

\section{Desenvolvimento}

\subsection{Atelectasia}

\subsubsection{Definição de Atelectasia}

Segundo Azeredo (2002, b), a atelectasia ou colapso pulmonar é uma complicação respiratória decorrente da obstrução de um brônquio, ou pulmão, seja por secreção ou ainda objeto ou corpo sólido, impedindo dessa forma a passagem do ar e levando à diminuição do número de alvéolos funcionantes.

\subsubsection{Fisiopatologia}

A fisiopatologia é basicamente quando há obstrução completa em um brônquio que supre de ar uma região normalmente ventilada do parênquima pulmonar, o gás nos alvéolos distais à obstrução é absorvido na circulação pulmonar. Esse processo de absorção geralmente não é imediato, levando várias horas. Uma vez que todo o gás alveolar tenha sido absorvido na circulação, os alvéolos, agora sem gás, colabam de forma semelhante a um balão que tenha perdido seu ar.

\subsubsection{Causas}

Azeredo (2002 b) define três fatores combinados ou independentes que contribuem para o desenvolvimento de uma atelectasia, que são: força inadequada de distensão pulmonar; obstrução das vias aéreas e insuficiência no surfactante.

\subsubsection{Classificação}

Azeredo (2002 a) classifica as atelectasias como: obstrutivas, compressivas, por contração e focal. As atelectasias obstrutivas são conseqüência de uma obstrução completa de uma via aérea, esse tipo de atelectasia é causado principalmente por secreções ou exudato excessivo dentro dos brônquios.

\subsubsection{Quadro Clínico}

Os sinais e sintomas das atelectasias variam com o tamanho da área pulmonar envolvida, o estado prévio de saúde do paciente e a duração do problema. A dispnéia é o mais comum dos sintomas associados à atelectasias, mas talvez não esteja presente se o paciente tiver um envolvimento pulmonar mínimo e tenha sido previamente saudável. O padrão respiratório do paciente costuma aumentar proporcionalmente com a área do pulmão envolvida com atelectasia.

\subsubsection{Apresentação Radiográfica}

A radiografia torácica é um instrumento de grande ajuda no diagnóstico de atelectasia. Ela pode revelar uma redução do volume pulmonar que não é clinicamente aparente. As alterações radiográficas que indicam colapso pulmonar total ou lobar são: deslocamento das fissuras, aumento da densidade pulmonar, aglomeração dos vasos, desvio do mediastino para o lado acometido; deslocamento hilar; elevação do diafragma para o lado afetado, costelas aproxima- 
das e hiper-insuflação compensatória do pulmão oposto, estas alterações não são vistas necessariamente em toda radiografia com diagnóstico de atelectasia.

\subsubsection{Ausculta Pulmonar}

A ausculta de sons respiratórios nem sempre ajuda a detectar um quadro de atelectasia. As crepitações inspiratórias são ouvidas em uma inspiração profunda e representam a abertura de regiões atelectasiadas, tais crepitações, em geral, são ouvidas inicialmente em regiões dependentes dos pulmões e talvez desapareçam se o paciente realizar severas respirações profundas. O murmúrio vesicular é diminuído ou abolido na região acometida.

\section{Tratamento da Atelectasia}

O tratamento da atelectasia inclui o clínico e o fisioterapêutico. No tratamento clínico, pode ser usado a broncoscopia como método de diagnóstico e terapêutico, pode ser usado também o tempo ou o grau da obstrução.(FARIA, 2002).

$\mathrm{O}$ tratamento deverá ser escolhido de acordo com a causa da atelectasia, determinando assim o objetivo para cada tipo de atelectasia.

\subsection{Tratamento Fisioterapêutico}

A fisioterapia respiratória pode atuar tanto na prevenção quanto no tratamento das atelectasias, utilizando-se de diversas técnicas e procedimentos terapêuticos. É importante ressaltar que, para se atingir resultados positivos, faz-se primordial um amplo estudo do quadro patológico apresentado pelo paciente, além de uma criteriosa avaliação das condições clínicas desse individuo e do traçado de um plano de tratamento condizente com suas necessidades atuais. O tratamento se modifica quando o paciente está em ventilação mecânica, ou respirando espontaneamente. $\mathrm{Na}$ atelectasia obstrutiva, a primeira escolha da fisioterapia é a desobstrução, na hipoventilação, será a melhora do padrão ventilatório.

\subsection{Técnicas Desobstrutivas}

\subsubsection{Drenagem Postural Brônquica Seletiva}

Segundo Tarantino (2002), a drenagem postural brônquica seletiva (DPBS) é uma das técnicas mais utilizadas na terapia de higiene brônquica. Ela envolve o uso da gravidade para auxiliar a movimentação da secreção pelo trato respiratório distal até o proximal, de onde pode ser eliminada pela tosse ou pela aspiração mecânica. Para se obter um bom resultado com essa técnica é necessário posicionar o paciente de forma adequada, levando em conta o brônquio de drenagem da região comprometida, e manter um bom nível de hidratação sistêmica.

\subsubsection{Terapia Manual Expiratória Manual por Pressão (TEMP)}

Consiste em uma variação da compressão torácica, na qual é realizado um aumento do fluxo expiratório no final da expiração, proporcionando um fluxo aéreo turbilhonar, favorecendo a mobilização de secreção brônquica.

\subsubsection{Compressão}

A técnica consiste em deprimir passivamente o gradil costal do paciente, além daquilo que ele consegue realizar ativamente, durante uma expiração normal ou forçada. Os objetivos são: desinsuflar o tórax e os pulmões, diminuir o volume residual, aumentar o volume corrente, aumentar a mobilidade da caixa torácica e estimular a expectoração.

\subsubsection{Vibração torácica}

A técnica consta em realizar uma vibração no tórax a fim de proporcionar o deslocamento das secreções brônquicas. A secreção brônquica tem uma característica interessante chamada de tixotropismo; quando aplicada uma vibração torácica à tendência do muco é se deslocar das paredes VA. (PRESTO e PRESTO, 2003)

\subsubsection{Ginga torácica}

O objetivo da ginga torácica é promover a mobilização de secreções broncopulmonares, através da movimentação seletiva das costelas e da geração de melhor fluxo expiratório. É geralmente utilizado após 
alongamento dos músculos inspiratórios, quando o retrocesso elástico da caixa torácica aumenta, tornandoa mais maleável e permitindo a aplicação de pressão sobre a parede torácica sem os efeitos de compressão dinâmica sobre as vias aéreas, o que contribuiria para o fechamento precoce destas.

O manuseio da ginga tem o objetivo de mobilizar seletivamente as costelas e deve ser feito de maneira alternada com suave pressão expiratória que orienta o movimento das costelas. O terapeuta deve posicionar as mãos na região inferior do tórax e aplicar pressão com direcionamento do movimento costal ora em um hemitórax ora no outro.

\subsubsection{Estímulo de Tosse}

As técnicas utilizadas pela fisioterapia podem ser realizadas de forma voluntária ou de forma a estimular uma tosse reflexa. Na tosse técnica, o fisioterapeuta pede ao paciente que realize uma tose voluntária, normal, com objetivo de expectorar.

Em pacientes comatosos e inconscientes, que estejam em ventilação mecânica, quando grande parte de seus reflexos estão abolidos ou diminuídos, a tosse está ausente, e necessita ser estimulada, usando das seguintes técnicas: tosse induzida pelo CUFF; tosse induzida por instilação traqueal; tosse induzida pelo ambú e tosse induzida pelo ventilador mecânico. (PRESTO e PRESTO, 2003).

\subsection{Terapia de Reexpansão Pulmonar}

\subsubsection{Direcionamento de Fluxo}

O objetivo dessa técnica seria direcionar o fluxo para um dos pulmões a fim de expandi-lo. (PRESTO e PRESTO, 2003). Realizamos a compressão em um dos hemitórax, no final da expiração, mantendo a compressão por até dez incursões respiratórias.

\subsubsection{Padrão Ventilatório com Inspiração Fracio- nada / Soluços Inspiratórios}

$\mathrm{Na}$ inspiração fracionada, o fisioterapeuta deve solicitar ao paciente que realize uma inspiração seguida de uma apnéia (pausa inspiratória) e assim sucessivamente até a capacidade pulmonar total (CPT) ser alcançada. Nos soluços inspiratórios, o fisiotera- peuta vai solicitar ao paciente que realize uma inspiração subdividida em inspirações curtas e sucessivas, sem apnéia, até alcançar a CPT. Nos soluços inspiratórios, a última incursão inspiratória deve ser realizada pela boca, de forma semelhante a um soluço.

As duas técnicas podem ser realizadas associadas à cinesioterapia de membros superiores e inferiores, não esquecendo de realizar uma pausa no movimento dos membros no momento da apnéia ou do soluço. É possível dividir a inspiração em vários números, porém, na prática, a fração utilizada em ambas as técnicas é até três (PRESTO e PRESTO, 2003).

\subsubsection{Sustentação Máxima da Inspiração}

A sustentação máxima da inspiração (SMI) é a técnica que pode ser utilizada associada a incentivadores respiratórios. A técnica consta em se manter uma apnéia (pausa ao final da inspiração) por, aproximadamente, 5 a 10 segundos. (PRESTO e PRESTO, 2003).

\subsubsection{Pressão Positiva Expiratória nas Vias Aéreas (EPAP)}

EPAP é um aparelho composto por uma máscara, uma válvula unidirecional e um gerador de PEEP, geralmente uma válvula spring loaded.

Os efeitos da EPAP são semelhantes aos efeitos da PEEP, a técnica pode ser usada com intuito de facilitar a desobstrução brônquica, por meio da ventilação de áreas hipoventiladas através da ventilação colateral e como forma de prevenção ao colapso das vias aéreas no final da expiração.

\subsubsection{Pressão Positiva Contínua nas Vias Aéreas (CPAP)}

É uma técnica que promove a manutenção de uma pressão positiva nas vias aéreas tanto na inspiração quanto na expiração. A CPAP aumenta a capacidade residual funcional e reduz o trabalho respiratório. A redução do trabalho respiratório é provavelmente devido ao aumento na complacência pulmonar, com o paciente respirando com volume pulmonar mais alto.

\section{Relato de Caso}


Paciente J.M., 78 anos, sexo masculino, foi encaminhado para internação na UTI do hospital VITA, no dia 26 de julho de 2006, com diagnóstico clínico de bronco aspiração. Acamado, com seqüela de AVE, gastrostomizado, apresentando tosse, tiragem intercostal e cianose generalizada, após ter sido alimentado por via oral. Foi realizado a EOT, com aspiração TOT, obtendo saída de grande quantidade de alimento. Foram realizadas avaliações clínicas e fisioterapêuticas com exames complementares, Raio X. No dia 03 agosto de 2006, às 9h, o paciente entrou em um quadro de falência respiratória, foram realizados exames complementares; em um exame de RX foi constatada uma atelectasia completa de pulmão esquerdo (Anexo I).

Mantido em ventilação mecânica, foi submetido a tratamento fisioterapêutico para reversão do quadro. O primeiro atendimento foi realizado ainda pela manhã, às $10 \mathrm{~h}$, mantendo os parâmetros do ventilador mecânico em PEEP de 10 cmH2O, volume corrente de $500 \mathrm{ml}$, e usando como base de tratamento, técnicas que estão descritas no protocolo de tratamento da equipe de fisioterapia do hospital VITA . O segundo atendimento foi realizado no mesmo dia, às 13h, quando os autores do trabalho acompanharam o tratamento, mantendo a mesma conduta do atendimento anterior. Após o segundo tratamento, foi realizado um RX de tórax para acompanhar a evolução do tratamento, quando se constatou a reversão da atelectasia, conforme RX (Anexo II).

\section{Considerações Finais}

Na fisioterapia respiratória, é importante destacar que para se ter resultado positivo no tratamento, deve-se avaliar as condições clínicas de cada paciente, traçando assim um tratamento condizente ao mesmo. Com base no que foi consultado nas literaturas, podemos concluir que a atelectasia representa uma das mais importantes indicações de fisioterapia respiratória em UTI. Ela é revertida totalmente em um curto espaço de tempo, comprovando assim a eficácia da atuação da fisioterapia na reversão das atelectasias em pacientes na UTI.

\section{Referências}

AZEREDO, C.A.C. Fisioterapia Respiratória. São Paulo: Panamed, 1984.

AZEREDO, C.A. Fisioterapia Respiratória Moderna. São Paulo: Manole, 2002a.

AZEREDO, C.A.C. Fisioterapia Respiratória Moderna. 4. ed. São Paulo: Manole, 2002b.

FARIA, E.C. Emergências Respiratórias. Rio de Janeiro: Editora de Publicações Biomédicas, 2002.

IRWIN, S.; TECKLIN, J.S. Fisioterapia Cardio Pulmonar. 2. ed. São Paulo: Manole, 1994.

KISNER, C.; COLBY, L.A. Exercícios Terapêuticos. Fundamentos e Técnicas. São Paulo: Manole, 1998.

KNOBEL, E.A. Terapia Intensiva. Pneumologia e Fisioterapia Respiratória. São Paulo: Atheneu, 2004.

PRESTO, B.L.V.; PRESTO, L.N. Fisioterapia Respiratória. Uma Nova Visão. Rio de Janeiro: Bruno Presto Editora, 2003.

STOLLER, J.K.; WILKINS, R.L.; SCANLAN, C.L. Fundamentos da Terapia Respiratória de Egan. 7. ed. São Paulo: Manole, 2000.

TARANTINO, A.B. Doenças Pulmonares. 5. ed. Rio de Janeiro: Guanabara Koogan, 2002. 


\section{Anexos}

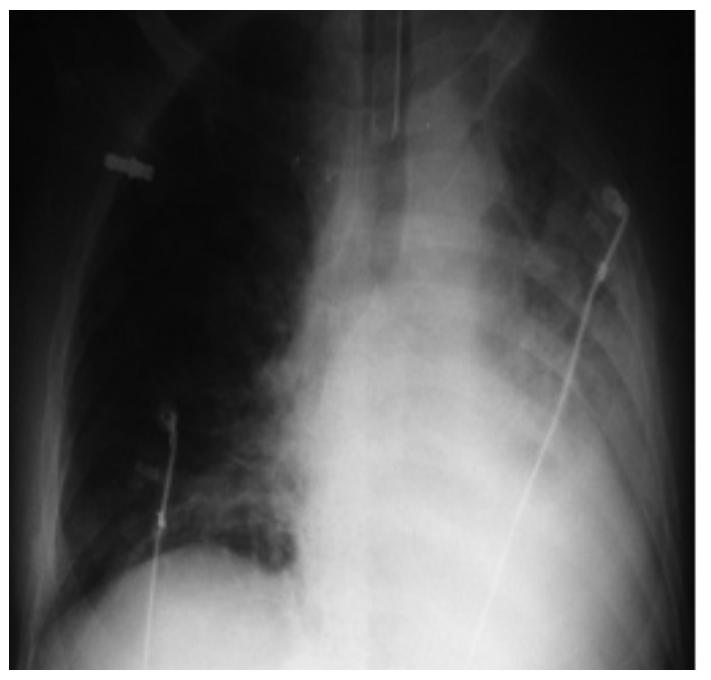

Antes da fisioterapia respiratória

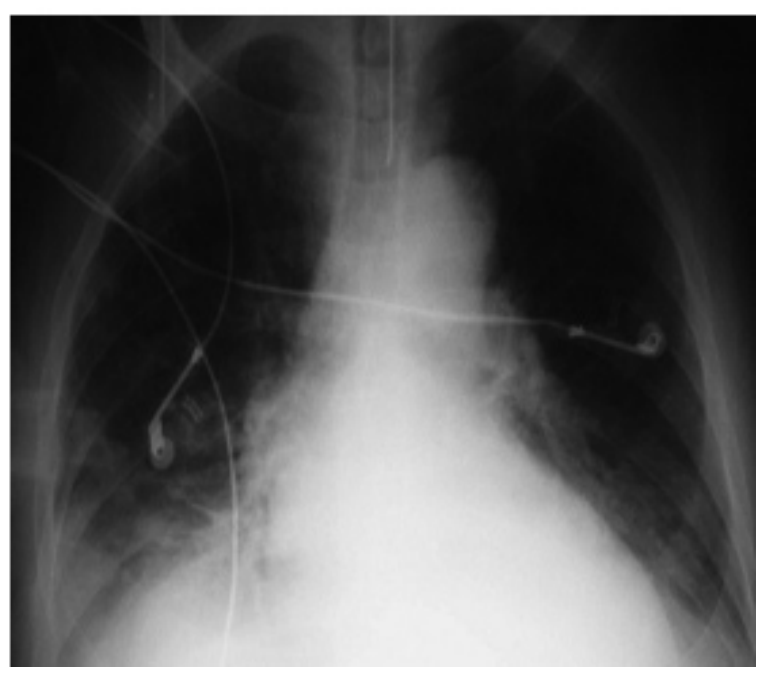

Após a fisioterapia respiratória

\section{Informações bibliográficas:}

Conforme a NBR 6023:2002 da Associação Brasileira de Normas Técnicas (ABNT), este texto científico publicado em periódico eletrônico deve ser citado da seguinte forma:

CUNHA, C. S.; TOLEDO, R. F.. Atuação da Fisioterapia na Reversão das Atelectasias: Um relato de caso na Unidade de Terapia Intensiva. Cadernos UniFOA, Volta Redonda, ano II, n. 4, agosto. 2007. Disponível em: $<$ http://www.unifoa.edu.br/pesquisa/caderno/edicao/04/81.pdf $>$ 